\title{
Luftverschmutzung fördert Schlaganfälle
}

\author{
Die Assoziation zwischen Luftverschmutzung und kardialen \\ Ereignissen ist hinreichend bekannt. Zur umweltbedingten \\ Inzidenz und Mortalität von Schlaganfällen gibt es allerdings \\ weniger konsistente Daten. Eine aktuelle Metaanalyse hat \\ nun eine enge zeitliche Assoziation mit der Luftschadstoff- \\ Konzentration gezeigt. \\ BMJ 2015; 350: h1295
}

Die Studienautoren wählten für die Analyse 94 Beobachtungsstudien nach vordefinierten Qualitätskriterien aus. Insgesamt wurden 6,2 Mio. Schlaganfall-Ereignisse in 28 Ländern ausgewertet. Endpunkte waren die Häufigkeit stationärer Einweisungen aufgrund von Schlaganfall sowie die Schlaganfall-Mortalität.

Beide Faktoren wurden jeweils in Abhängigkeit von der Luftschadstoff-Exposition innerhalb der vorangegangenen 7 Tage untersucht.
Die Endpunkte waren assoziiert mit der

- CO-Konzentration: relatives Risiko 1,015 pro 1 part per million (ppm; 95\%-Konfidenzintervall 1,004-1,026),

- der $\mathrm{SO}_{2}$-Konzentration:1,019 pro 1 part per billion (ppb; 1,011-1,027),

- $\mathrm{NO}_{2}$-Konzentration: 1,014 pro $10 \mathrm{ppb}$ (1,009-1,019),

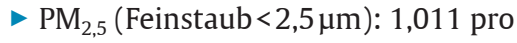
$10 \mu \mathrm{g} / \mathrm{m}^{3}(1,011-1,012)$,

- $\mathrm{PM}_{10}$ (Feinstaub<10 $\left.\mu \mathrm{m}\right):$ 1,003 pro $10 \mu \mathrm{g} / \mathrm{m}^{3}(1,002-1,004)$ und

- dem Ozon-Wert: 1,001 pro $10 \mathrm{ppb}$ $(1,000-1,002)$.
Die deutlichste Assoziation bestand am Tag der Exposition. Die Auswirkungen der $\mathrm{PM}_{2,5}$-Belastung waren am stärksten und hielten am längsten an. Ein Grund hierfür könnte sein, dass sehr kleine Feinstaubpartikel direkt von der Lunge in den Blutkreislauf übertreten können. Die Forscher vermuten, dass die unmittelbaren Effekte der Luftverschmutzung über das vegetative Nervensystem und Veränderungen der Endothelfunktion verursacht werden. Hinsichtlich hämorrhagischer Schlaganfälle ist die Aussagekraft der Ergebnisse allerdings eingeschränkt, da diese deutlich seltener auftreten als ischämische Apoplexe.

Fazit
Der Schadstoffgehalt der Luft und die
Häufigkeit von Krankenhauseinweisun-
gen bzw. Todesfällen in Folge von Apo-
plexen hängen zeitlich eng zusammen.
Gesellschaftliche und politische An-
strengungen zur Reduktion der Luftver-
schmutzung könnten dazu beitragen,
die Inzidenz von Schlaganfällen zu ver-
mindern, so die Autoren.

Dr. Peter Pommer, Oberammergau

Eine Studie, die E-Zigaretten mit Nikotinpflastern verglich, fand keine statistisch signifikanten Unterschiede bei der Tabakabstinenz nach 6 Monaten (RR 1,26 [0,68$2,34]$ ). Allerdings konnten deutlich mehr Studienteilnehmer im E-Zigarettenarm die Anzahl der Zigaretten am Tag reduzieren (61 vs. $44 \%$ der Teilnehmer bei Pflastern, RR 1,41 [1,20-1,67]). Keine der Studien berichtete von ernsthaften Nebenwirkungen. Wegen der geringen Anzahl der Studien bewerten die Autoren die Qualität der Evidenz nach GRADE als niedrig.

\section{Fazit}

Die beiden Studien deuten nach Angaben der Autoren darauf hin, dass die Verwendung von E-Zigaretten im Vergleich zu Placebo die Chancen auf eine Tabakabstinenz erhöht. Im Vergleich mit Nikotinpflastern lässt sich aufgrund der geringen Probandenzahl keine Aussage treffen. Da die Qualität der Evidenz wegen der geringen Studienzahl niedrig ist, sind weitere Untersuchungen notwendig.

Dr. Peter Pommer, Oberammergau 